flushed with $250 \mathrm{~mL}$ of saline. After flushing, frank hematuria subsided; however, blood tinged urine continued to drain in the postoperative period. Urosurgical workup was done postoperatively. USG KUB was inconclusive. CT urogram was done, revealing retroperitoneal hematoma extending up to right psoas muscle; right perirenal hematoma with renal contusion and clots in urinary bladder. Patient was managed conservatively.

Conclusion: Spine injury, renal injury and retroperitoneal hematoma are common occurrence following vertical deceleration injury due to fall from height. High index of suspicion must be maintained even if initial imaging misses these injuries. Intraoperative hematuria in this case was due to dislodgement of clots in kidney, following prone positioning.

\section{A023 Airway Management in Frontonasal Encephalocele} Shalaka T., ${ }^{1}$ Karen R. Lionel, ${ }^{1}$ Ramamani Mariappan ${ }^{1}$ ${ }^{1}$ Department of Anaesthesia, Christian Medical College (CMC), Vellore, Tamil Nadu, India

Background: Frontonasal encephalocele is a congenital malformation, involving the herniation of intracranial contents from foramen cecum at the junction of nasal and frontal bones. Apart from inherent anesthetic implications of pediatric patients and associated systemic abnormalities; airway management presents an anesthetic challenge.

Case Description: A17-month-old male, weighing $6.2 \mathrm{~kg}$ admitted with a large frontonasal $(7 \mathrm{~cm} \times 5 \mathrm{~cm})$, encephalocele at glabela, extending down from right nostril to upper lip, planned for bifrontal craniotomy and excision. During preanesthetic evaluation, difficult mask ventilation was anticipated. Since there were no signs of raised ICP, he was premedicated with trichloryl which enabled us for smooth induction. The child was asleep on arrival to OR, steal induction was performed with sevoflurane, and an i.v. cannula was secured. Ability to mask ventilate was confirmed by placing an adult no. 5 mask over the face rotated 180 degrees to fit the contour of the face which gave a good seal along the frontal, zygomatic bones, and mandible. Measures were taken to avoid direct pressure over the eyes by applying soft gauze pads. Direct laryngoscopy with McIntosh blade size no. 2 revealed Cormack-Lehane grade 1. Intraoperative period was uneventful. He was extubated and shifted to the NICU.

Conclusion: Patients with frontonasal encephalocele may present with difficulty in mask ventilation. Conventional mask holding can cause potential rise in ICP due to compression of the sac during mask holding and can cause rupture of the sac. Hence the anesthetist must have a rescue plan in the event of not being able to gas induce or mask ventilate.

\author{
A024 Symptomatic Pneumocephalus following Spine \\ Surgery: A Case Report and Literature Review \\ Sriram Ravinutala, ${ }^{1}$ Harshal Dholke, ${ }^{1}$ Sai K. Savarapu ${ }^{2}$ \\ ${ }^{1}$ Department of Anesthesiology, Krishna institute of Medical \\ Sciences (KIMS), Secunderabad, Telangana, India \\ 2Department of Neurosurgery, Krishna institute of Medical \\ Sciences (KIMS), Secunderabad, Telangana, India
}

Background: Pneumocephalus, though frequently seen after cranial trauma and surgery, is an uncommon complication following spine surgery.

Case Description: We present the case of a 20-year-old male patient on whom a D10-L1 laminectomy was performed for the excision of an intradural tumor. The patient experienced a delayed emergence from anesthesia followed by tonic-clonic seizures after extubation. He was stabilized and the seizures were controlled while altered sensorium persisted into the early postoperative period. Imaging studies showed the presence of a pneumocephalus which was managed conservatively in the neurosurgical intensive care unit.

Conclusion: Through this presentation, we attempt to throw light on the mechanisms of development of pneumocephalus after surgical interventions on the spine, discuss the clinical presentation and diagnosis, and suggest possible measures to prevent development of this rare yet clinically significant condition.

\section{A025 Role of Transesophageal ECHO in Ventriculoperitoneal Shunt Surgery for a Child with Dandy-Walker Malformation: A Case Report Preethi Kuryan, ${ }^{1}$ Edmond J. Gandham, ${ }^{1}$ \\ Ramamani Mariappan ${ }^{1}$ \\ ${ }^{1}$ Department of Anaesthesia, Christian Medical College (CMC), Vellore, Tamil Nadu, India}

Background: Dandy-Walker malformation (DWM) is a congenital malformation, characterized by enlargement of the posterior fossa, cystic dilatation of the fourth ventricle, and agenesis/hypoplasia of the vermis. We would like to report the role of transesophagealecho (TEE) for confirming the position of distal tip during ventriculoatrial (VA) shunt insertion.

Case Description: A 2-year-old boy weighing $7.5 \mathrm{~kg}$ was admitted with diagnosis of DWM with shunt dysfunction following a cystoperitoneal shunt and was planned for insertion of VA shunt. Since, the child had features of raised ICP, an intravenous access was secured after applying the EMLA cream. Standard monitors were applied, the child was intubated after fentanyl, propofol, and atracurium using 5-size uncuffed tube. Anesthesia was maintained with propofol infusion till the insertion of VA shunt to avoid further increase in ICP. TEE probe (pediatric) was inserted for confirming the optimal position of the distal tip and to detect air embolism. Child was hydrated well with $20 \mathrm{~mL} / \mathrm{kg}$ of RL to make the internal jugular vein prominent and to avoid air entrainment while inserting the distal tip into the atrium. All catheters were flushed with saline and 10-degree head down was given while inserting the distal tip. The distal tip was placed at the mid atrial level and its correct placement was confirmed by transesophageal echo.

Conclusion: Accurate placement of distal tip of the VA shunt is crucial to ensure long-term shunt function, prevention of cardiac arrhythmias, and myocardial damage. TEE is useful as it provides a real time image, enables continuous monitoring of tip, and occurrence of air embolism. 